\title{
PREFACE
}

\section{Preface to the special issue on 'Disordered serendipity: a glassy path to discovery'}

To cite this article: Andrea Cavagna et al 2020 J. Phys. A: Math. Theor. 53500301

View the article online for updates and enhancements.

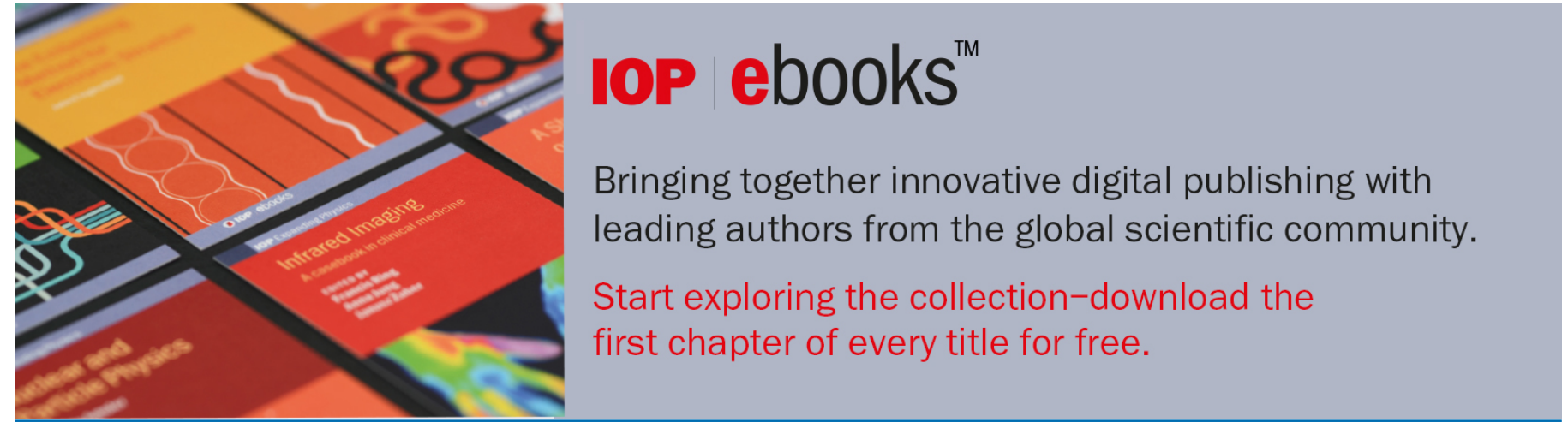

This content was downloaded from IP address 141.108 .46 .2 on 10/10/2021 at $16: 39$ 


\section{Preface to the special issue on 'Disordered serendipity: a glassy path to discovery'}

Giorgio Parisi belongs to a rare class of universal scientists. For fifty years, his large breadth of interests and creative intuition has led to new seminal ideas in far-away areas of science. In his scientific career he addressed topics as diverse as particle physics, field theory, statistical mechanics, fluid dynamics, condensed matter, numerical simulations and the constructions of scientific computers.

The trans-disciplinary approach of statistical physics to complex systems has been one of the long lasting interests of Parisi, who contributed to the theory of neural networks and the immune system, surface growth, optimization and computational complexity and the collective movement of groups of animals. His work has had a big impact in all the fields he touched.

A single work that represents a genuine breakthrough, is the replica symmetry breaking approach to disordered systems and its twin formulation, the cavity method. These methods led to a new understanding in the whole field of complex systems and keep generating new knowledge and stimulating multidisciplinary research.

In 1979 Parisi found the exact solution of the Sherrington-Kirkpatrick spin glass through an astonishing analysis of the permutation group of $n$ elements in the limit $n \rightarrow 0$. The effort to interpret his replica symmetry breaking (RSB) led him and his collaborators Mézard and Virasoro to predict the existence of a new surprising phase, with broken ergodicity, hierarchically organised pure states and non-trivial fluctuations of intensive thermodynamic quantities.

The mathematisation of these pioneering results required 30 years of efforts and mathematicians are now discovering vast extensions of these concepts. The impact in physics and other areas of science was immediate.

The newly gained confidence in the replica method allowed in the 80's to solve Hopfield model of a neural network and a myriad of applications in neural networks and machine learning appeared.

In the same years, the RSB based random first order transition theory (RFOT) for structural glasses was proposed. From the 90 's the deeply innovative extensions of the cavity and replica method to models on Bethe lattices, led to spectacular advances in computer science. Parisi with Mézard and Zecchina could exactly find the satisfiability threshold in the celebrated random K-Sat model, and, most interestingly, exploited this exact solution to build a new family of algorithms that improved the state of the art by several orders of magnitude.

More recently the RSB approach allowed the exact description of glassy phases of particles in the limit of high dimension. This is the last step in Parisi's long quest for a first principle theory of structural glasses. This solution provides the microscopic foundation of RFOT theory. At the same time it proposes a picture that goes much beyond, predicting the existence of a new fundamental glass-to-glass transition at low temperature where the glass becomes marginally 
stable and ungapped excitations appear. Numerical evidence in favour of the presence of this transition in finite dimension has been found by Parisi and collaborators. The most spectacular tested consequence of the theory is a universal description of jammed states of hard spheres, which accounts for the behaviour found in numerical simulations (also by the group of Parisi) in spatial dimension $D$ spanning from 2 to 8 . While the infinite dimensional solution will remain a cornerstone in the theory of the glasses the derivation of all its implication is only at the beginning.

The present special issue celebrates Giorgio Parisi's 70th birthday, and tries to give an overview of the current state of research in the fields of statistical mechanics and interdisciplinary applications which have been marked by Giorgio Parisi's seminal contributions. The manuscripts accepted for publication in this special issue do indeed cover a broad range of subjects, but they share the same theoretical approach based on the techniques pioneered by Giorgio Parisi.

\section{Andrea Cavagna}

Istituto dei Sistemi Complessi, Consiglio Nazionale delle RIcerche, Via dei Taurini 19, 00185

Roma, Italy

Dipartimento di Fisica, Università “'La Sapienza', P.le A. Moro 5, 00185, Rome, Italy

Silvio Franz* (D)

Université Paris-Saclay, CNRS, LPTMS, 91405, Orsay, France

\section{Irene Giardina}

Dipartimento di Fisica, Università 'La Sapienza', P.le A. Moro 5, 00185, Rome, Italy CNR-ISC, Sapienza unit and INFN, Sezione di Roma1, P.le A. Moro 5, 00185, Rome, Italy

\section{Luca Leuzzi}

CNR-Nanotec, Rome unit, P.le A. Moro 5, 00185, Rome, Italy

Dipartimento di Fisica, Università 'La Sapienza', P.le A. Moro 5, 00185, Rome, Italy

\section{Andrea Maiorano}

Dipartimento di Biotecnologie, Chimica e Farmacia

Università degli Studi di Siena, 53100 Siena; Insituto de Biocomputación y Física de Sistemas Complejos, 50018 Zaragoza, Spain

\section{Enzo Marinari}

Dipartimento di Fisica, Università “La Sapienza", P.le A. Moro 5, 00185, Rome, Italy

INFN, Sezione di Roma1, and CNR-Nanotec, Rome unit, P.le A. Moro 5, 00185, Rome, Italy

\section{Federico Ricci-Tersenghi 1}

Dipartimento di Fisica, Università “La Sapienza”, P.le A. Moro 5, 00185, Rome, Italy

INFN, Sezione di Roma1, and CNR-Nanotec, Rome unit, P.le A. Moro 5, 00185, Rome, Italy

\section{Tommaso Rizzo 10}

ISC-CNR, Università di Roma “'La Sapienza”, 00185 Rome, Italy

Dipartimento di Fisica, Università di Roma 'La Sapienza', 00185 Rome, Italy

\footnotetext{
* Author to whom any correspondence should be addressed.
} 
Francesco Zamponi ${ }^{\circ}$

Laboratoire de Physique de l'Ecole Normale Supérieure, ENS, Université

PSL, CNRS, Sorbonne Université, Université de Paris, F-75005 Paris , France

E-mail: silvio.franz@u-psud.fr

\section{ORCID iDs}

Silvio Franz (iD) https://orcid.org/0000-0001-8300-8443

Luca Leuzzi@ https://orcid.org/0000-0002-5971-7191

Enzo Marinari(D) https://orcid.org/0000-0002-3464-4133

Federico Ricci-Tersenghi (D) https://orcid.org/0000-0003-4970-7376

Tommaso Rizzo@ https://orcid.org/0000-0001-5902-0565

Francesco Zamponi(i) https://orcid.org/0000-0001-9260-1951 